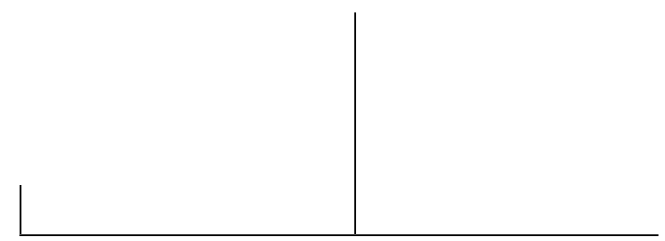

Rev. Latinoam. Psicopat. Fund., São Paulo, 16(3), 438-450, set. 2013

\title{
Apoio matricial em saúde mental: fortalecendo a saúde da família na clínica da crise*1
}

O objetivo consiste em abordar o apoio matricial em saúde mental às equipes de Saúde da Família (SF) e a sua relação com as situações de crise em saúde mental. Trata-se de uma pesquisa- ação realizada com equipes de SF e com um CAPS III, na Rocinha, Rio de Janeiro. Participaram profissionais de diferentes categorias em grupos operativos de reflexão e os dados foram examinados pela análise de conteúdo. É destacada a importância de envolver a SF no que se refere à clínica da crise.

Palavras-chave: Serviços de Saúde Mental, atenção primária à saúde, apoio matricial, crise

${ }^{*}$ A reflexão que este artigo propõe é parte integrante da dissertação de mestrado do Programa de Pós-Graduação em Psicologia Clínica e Cultura (PsiCC) da Universidade de Brasília (UnB), intitulada "Análise da implantação do Apoio Matricial em Saúde Mental entre CAPS e Equipes de Saúde da Família: trilhando caminhos possíveis”. Dissertação defendida e aprovada em julho de 2011 na Universidade de Brasília (UnB) pela primeira autora sob orientação do segundo autor.

*22Universidade de Brasilia - UnB (Brasília, DF, Brasil).

${ }^{* 3}$ Universidade de Brasilia - UnB (Brasília, DF, Brasil). 
Este artigo discute o apoio matricial em saúde mental às equipes de Saúde da Família (SF) e a sua relação com as situações de crise. O apoio matricial em saúde mental é uma prática em que profissionais especialistas em saúde mental oferecem suporte a equipes de SF com o objetivo de ampliar a sua resolubilidade e produzir maior responsabilização no acompanhamento e atendimento das pessoas em sofrimento psíquico, rompendo com a lógica dos encaminhamentos indiscriminados e ampliando a clínica da SF.

$\mathrm{O}$ apoio matricial, ou matriciamento, é utilizado ou solicitado quando a equipe SF encontra limites ou dificuldades na condução dos casos clínicos e na direção ou planejamento de atividades específicas. Este facilita a troca de opinião entre os profissionais, convidando-os a elaborar conjuntamente projetos terapêuticos singulares, baseados no diálogo e no compromisso com a saúde dos usuários (Campos, 1999; Campos \& Domitti, 2007). Objetiva ampliar o campo de atuação e qualificar as ações das equipes de SF, inclusive nas situações de crise.

A crise, numa perspectiva psicológica, pode ser entendida como sendo uma manifestação súbita de uma ruptura de equilíbrio preexistente. Nesse sentido, os momentos da vida considerados como geradores de crise, como adolescência, casamento, separação, perda de emprego não levam necessariamente a pessoa à crise. Pode-se entender que a situação de crise é desencadeada quando o sujeito, ao necessitar dar respostas frente a algumas situações da vida, não possui recursos emocionais para tal, gerando um rompimento de seu equilíbrio psíquico (Costa, 2006). O contexto de crise, nesse sentido, "é aquele cujo aparecimento causa desequilíbrio psíquico, no qual o sujeito se encontra desprovido das competências que o levam a uma reacomodação às situações de conflito" (Carvalho \& Costa, 2008, p. 154). 
Também, a crise está relacionada com os contextos reais da vida do sujeito, correspondendo "a uma complexa situação existencial" (Basaglia, 1991, p. 55). Desta forma, é necessário colocá-la no interior de uma série de nexos e fazer uma leitura da situação, para compreendê-la. Segundo Nicácio \& Campos (2004), a crise torna-se um evento histórico, que se coliga com a história do sujeito. Sendo assim, "a complexidade das situações de crise não pode ser lida como um dado isolado e a própria compreensão é redimensionada em processo na continuidade da relação" (p. 76).

Os Centros de Atenção Psicossocial (CAPS) são considerados estratégicos para o cuidado da crise. Serviços da Reforma Psiquiátrica brasileira, os CAPS apontam para a possibilidade de organização de uma rede substitutiva ao hospital psiquiátrico no país. Os CAPS III são serviços de maior complexidade, com funcionamento 24 horas, durante todos os dias, inclusive feriados e finais de semana. Na rede de saúde mental, os CAPS III acolhem a crise, oferecem acolhimento noturno, proporcionam atenção integral e buscam reduzir as internações psiquiátricas (Brasil, 2004).

Já os serviços de Atenção Primária à Saúde (APS) se constituem na porta de entrada preferencial do Sistema Único de Saúde (SUS), onde são identificadas e coordenadas as respostas para as necessidades de saúde das pessoas, suas famílias e comunidades. No Brasil, a SF, estratégia prioritária de reorganização da APS, é responsável por um dos mais significativos movimentos de ampliação do acesso à saúde. A SF é operacionalizada mediante a implantação de equipes multiprofissionais, que possuem a responsabilidade de prestar atenção à saúde a uma população de um território adscrito, tendo como máximo recomendado o equivalente a quatro mil pessoas sob sua responsabilidade (Brasil, 2011). No que se refere às pessoas em situação de crise, ainda é necessário investir em estratégias que incluam o acolhimento, avaliação, intervenções breves no cotidiano da SF. Para tal, o apoio matricial em saúde mental é considerado uma prática que auxilia as equipes de SF na avaliação e condução de casos e situações de crise.

$\mathrm{O}$ intuito desse estudo é elucidar as dificuldades e potencialidade da SF na atenção à crise e o apoio matricial em saúde mental como uma estratégia para fortalecer a prática da SF na atenção psicossocial. Nesta pesquisa, aborda-se o apoio matricial realizado pelos profissionais dos CAPS às equipes de SF.

\section{Percurso metodológico}

Os dados desta pesquisa foram obtidos através de três grupos operativos realizados com profissionais da SF e de três grupos operativos que integraram 
profissionais da SF e de um CAPS III. Participaram seis profissionais de nível superior da SF, dentre eles enfermeiros e dentistas e 15 profissionais do CAPS III de diferentes categorias profissionais - Psicologia, Nutrição, Musicoterapia, Enfermagem, Terapia Ocupacional e Técnicos de Enfermagem.

Trata-se de uma pesquisa qualitativa, de cunho exploratório, estruturada na modalidade da pesquisa-ação. Por seu duplo objetivo de modificar a realidade e produzir conhecimentos, a pesquisa-ação favorece que participantes e pesquisadores se envolvam de modo cooperativo e participativo, produzindo novas reflexões e práticas (Barbier, 2002; Thiollent, 1996).

Os grupos operativos de reflexão consistiram na estratégia escolhida para a realização da pesquisa-ação. As técnicas utilizadas foram construídas conjuntamente entre pesquisador e participantes, optando-se por leitura e debate de textos, discussão de casos clínicos e de experiências de apoio matricial, reflexões em grupo, questionários e avaliações dos encontros.

Utilizou-se a análise de conteúdo e sua técnica temática como forma de tratamento das informações. Segundo Bardin (1977), a análise de conteúdo consiste num conjunto de técnicas de análises de comunicações, utilizando-se de procedimentos sistemáticos e objetivos de descrição do conteúdo das mensagens. Para a análise de conteúdo realizou-se a pré-análise, na qual se fez a escolha do material coletado e a leitura flutuante. Após, foi realizada a categorização, com os temas que tiveram a mesma significação (Franco, 2003).

Foi realizado um encontro com os profissionais que participaram da pesquisa para apresentação e discussão dos resultados, com a finalidade de contribuir com a análise dos dados e com a disseminação, em serviço, dos seus achados. A pesquisa foi submetida e aprovada pelo Comitê de Ética em Pesquisa da Secretaria Municipal de Saúde e Defesa Civil do Rio de Janeiro.

\section{Análise e discussão dos resultados}

Nos resultados sobre a temática apoio matricial e as situações de crise, duas categorias foram evidenciadas: 1) SF e as situações de crise; 2) Integração entre CAPS e SF: considerações sobre os encaminhamentos das situações de crise.

\section{Saúde da Família e as situações de crise}

Nos grupos operativos, formados pelos profissionais da SF, foi discutido um caso clínico de um paciente e sua família que procuram a equipe de SF para aten- 
dimento de uma situação de crise do tipo psicótica. Os dados da pesquisa indicaram a coexistência dos dois modos de cuidado, asilar e psicossocial, na atenção em saúde mental promovida pelas equipes de SF.

De acordo com Costa-Rosa (2000), o modo asilar corresponde ao paradigma clássico da atenção em saúde mental, centra o cuidado nos aspectos orgânicos, na doença e no medicamento. Já o modo psicossocial, situado em contraposição ao asilar, tem como seu objeto a existência-sofrimento em sua relação com o corpo social e como objetivo a emancipação social do sujeito, sendo decisiva a sua participação no tratamento. Busca uma mudança na forma de relação dos usuários consigo mesmos, com o medicamento, com a família, com o tratamento e com os próprios profissionais da saúde.

No que se refere ao modo psicossocial, nas falas dos participantes da SF foram evidenciadas concepções que apontam para o reconhecimento da importância do vínculo e da confiança entre profissionais e usuários na realização das ações de saúde mental. Além de serem identificados aspectos que sinalizam uma concepção ampliada do processo saúde-doença e o reconhecimento da importância da escuta e da troca entre os usuários como promotores de saúde mental.

Neste sentido, Basaglia (1991) aponta que as relações de cumplicidade e confiança entre profissionais e pacientes representam atos terapêuticos em si, pois a escuta favorece a colocação da realidade do paciente numa perspectiva histórica, explicitando as contradições e os conflitos que se mobilizam. Estas relações estão voltadas à recomposição da identidade do paciente e da ruptura de relações que precedeu a crise. Ainda, comunicam a disponibilidade do serviço em direção ao paciente, de maneira a não confirmar um rótulo e a posterior perda de poder, como a internação por si mesma.

Ao mesmo tempo em que as concepções e práticas dos profissionais apontaram para o modo psicossocial na atenção em saúde mental, emergiram falas associadas a concepções e intervenções asilares. O encaminhamento para a internação psiquiátrica foi uma das primeiras alternativas pensadas pelos profissionais quando discutido o caso do paciente em crise do tipo psicótica que procura a equipe de SF. Em caso de negativa do paciente em buscar o serviço indicado, os profissionais da SF propuseram intervenções que ignoram o sujeito, como por exemplo, a imposição do medicamento e a colocação à força em ambulância para o transporte até a emergência psiquiátrica. A medicação apareceu como procedimento necessário nesses momentos.

Eu mesma iria chamar a ambulância para dar uma injeção para que ele pudesse ser levado para emergência psiquiátrica, porque como que eu vou deixar ele sair [...]. Ele não quer ir, eu estou mandando ele ir. (Enfermeira) 


\section{SAÚDE MENTAL}

Como não tem como prender ele aqui eu vou prender ele em algum lugar, vou chamar alguém pra isso. Eu vou prender ele em alguma emergência psiquiátrica, mas pra casa ele não vai. (Enfermeira)

O que vocês estão falando é do surto, normalmente o que acontece não é você tentar uma conversa com o paciente. Na maioria dos casos você vai lá e parte pro procedimento, medicação. (Enfermeira)

Sabe-se que um sujeito em crise do tipo psicótica, em risco de auto ou heteroagressão, necessita especial atenção tendo direito à proteção e resguardo, não podendo ser negligenciado, bem como o medicamento pode ser de extrema importância para diminuir a angústia e aliviar a dor. O que se destaca é que os dados indicam que a crise parece assustar os profissionais da SF. Nessa situação, evidencia-se a fragilidade da contratação das intervenções, tanto com o paciente quanto com a família, característica do modo asilar em que pouca ou nenhuma consideração é dada à existência do sujeito e que o tratamento é focado na prescrição do remédio (Costa-Rosa, 2000).

No que se refere à medicação, há a necessidade do não abafamento da crise com grande quantidade de remédios, possibilitando o tratamento e acompanhamento do sujeito que sofre. A prescrição inadequada de medicamentos pode ser prejudicial aos usuários, principalmente quando é usada no sentido da não responsabilização do sujeito pelo seu problema e quando é utilizada como uma forma de apaziguamento da pessoa, deixando-a prostrada e passiva, impedindo o seu progresso no tratamento (Onocko Campos \& Gama, 2001; 2010).

Nas falas dos participantes da SF, a avaliação médica na crise mostrou-se fundamental. "Ele vai ter que passar por uma avaliação médica, mesmo não sendo psiquiatra" (Enfermeira); "O médico vai ajudar muito na conduta clínica com esses pacientes" (Enfermeira).

O médico, como parte de uma equipe de saúde, possui especificidades e atribuições, sendo considerado peça fundamental para o funcionamento da engrenagem da SF. Porém, quando lhe é conferido o poder de soberania, os demais profissionais podem ficar na sua dependência, eximindo-se de realizar práticas como o acolhimento, avaliação da situação de crise e as intervenções breves.

Acredita-se que são importantes investimentos no fortalecimento da saúde mental na SF, com a inclusão da dimensão do cuidado frente às crises. Sabe-se que em muitos casos a atenção a alguns tipos de crises vai demandar cuidados intensivos, mas crises mais brandas podem ser cuidadas pela SF, e as mais graves podem ser avaliadas, acolhidas e encaminhadas de forma responsável, favorecendo o vínculo, a responsabilização e a coordenação do cuidado, a partir da SF.

Enfim, tais resultados indicam a importância de colocar em pauta a discussão sobre o papel da SF nas situações de crise e as intervenções possíveis de se- 
rem realizadas nesse nível de atenção. Para tal, é fundamental fortalecer o modo psicossocial na atenção em saúde mental dos profissionais da SF.

\section{Integração entre CAPS e SF: considerações sobre os encaminhamentos das situações de crise}

Nos grupos mistos, em que ocorreu a integração entre os profissionais da SF e do CAPS III, emergiu o tema referente a como proceder para encaminhar, a partir da SF, os casos para o CAPS III. Os profissionais da SF questionaram sobre quais os critérios, qual seria a forma, quais seriam os casos encaminhados e quais seriam discutidos nos encontros de apoio matricial. "Como que a gente encaminha, quando não encaminha, quando que a gente deixa o paciente na Clínica da Família?”. (Enfermeira - SF).

Tais questões remeteram o grupo à reflexão sobre a importância da discussão dos casos, a partir do apoio matricial. O grupo refletiu que a discussão dos casos clínicos auxilia a equipe de SF na condução clínica e, com isso, alguns encaminhamentos tornam-se desnecessários.

Mas essa é a questão do matriciamento: ele não vai deixar de ser um paciente de vocês. Você vai trazer, muitas vezes nem o paciente, você vai trazer o caso, nós vamos discutir o caso e vamos orientar, aí você vai levar e a gente vai trabalhar a evolução. (Técnico de Enfermagem - CAPS)

Foi dialogado sobre a importância de discutir os casos com os matriciadores, antes dos encaminhamentos. A discussão dos casos fortalece a melhor avaliação da gravidade, dos riscos e vulnerabilidades e os encaminhamentos serão feitos quando houver legítima necessidade para tal. "Antes de qualquer encaminhamento, você tem que ter uma discussão com a equipe de matriciadores. A não ser que seja uma crise." (Técnico de Enfermagem - CAPS).

Ressalta-se que é importante romper a associação habitualmente feita entre crise e periculosidade, que produz temor nos profissionais em se aproximar e escutar o paciente e o contexto em que esta crise emergiu. Uma crise mais branda pode ser tratada pela própria equipe de SF, desde que o paciente seja atendido cotidianamente pelo seu profissional de referência e possa procurá-lo sempre que sentir necessidade; outra, mais grave, pode necessitar de permanência no CAPS. Contudo, em hipótese alguma, pode-se abrir mão daquilo que pareça realmente essencial para um tratamento adequado para cada caso e para a proteção do paciente (Lobosque, 2007).

Porém, percebeu-se nos dados da pesquisa a referência de que os casos em crise podem ser encaminhados para o CAPS sem necessariamente passar pela dis- 
cussão do apoio matricial, o que de fato pode acontecer, desde que a SF realize a avaliação do caso e identifique que esta é a melhor conduta a ser seguida. Caso contrário, uma avaliação conjunta da situação, entre apoiador matricial e SF, pode ser o caminho a ser trilhado, tanto para fortalecer a SF quanto para propor uma conduta clínica que atenda a necessidade do paciente naquele momento, e indique o melhor percurso terapêutico a ser seguido.

Esta discussão em grupo indicou para a SF que o CAPS III é um local para o acolhimento da crise, e que não é necessário o encaminhamento direto para a emergência psiquiátrica, lugar identificado pelos profissionais da SF nas discussões do grupo homogêneo. Além disso, sinalizou a importância da escuta dos casos em crise, antes do encaminhamento. De acordo com Basaglia (1991), a escuta favorece a colocação da crise em uma perspectiva histórica, tornando mais conscientes o paciente e os seus familiares, e explicitando as contradições e os conflitos que se mobilizam neste momento de vida.

Porém, acredita-se ser fundamental aprofundar a discussão sobre o papel da SF nos atendimentos da crise, elucidando critérios de avaliação de risco, vulnerabilidade e urgência em saúde mental, a fim de que a SF se aproprie de tais abordagens e desenvolva a capacidade de avaliar os casos antes de encaminhar os pacientes. Possibilitando, assim, que sejam identificadas quais situações de crise podem ser cuidadas na SF e quais necessitam de um cuidado mais intensivo. Mas, para isso, este estudo sinalizou que um dos primeiros passos consiste na desmistificação de que a pessoa em crise é perigosa e de que a crise é um evento isolado na vida dos sujeitos.

Sendo assim, os grupos mistos permitiram a reflexão sobre aspectos fundamentais na organização e concepção do apoio matricial, como o papel da SF no acolhimento da crise e da redução dos encaminhamentos indiscriminados. Mas, para isso, é necessário que o apoio matricial seja colocado em pauta como uma ação importante no processo de cuidado em saúde mental.

\section{Considerações finais}

Esta pesquisa sinalizou a coexistência dos modos asilar e psicossocial nas práticas de cuidado das equipes de SF. No que se refere ao modo psicossocial, identificou-se que a SF valoriza o vínculo entre profissionais e pacientes, a escuta, as trocas entre os usuários e as práticas que contemplam a compreensão do contexto em que as pessoas estão inseridas. Mas, também foram identificadas concepções vinculadas ao modo asilar, em que foram indicadas práticas que incentivam a hospitalização, a falta de contratualização com os usuários e fami- 
liares, a dependência do saber médico e da medicação. Dessa forma, acredita-se ser importante que as ações de apoio matricial invistam no fortalecimento do modo psicossocial, auxiliando os profissionais da SF no rompimento com as atitudes ainda voltadas ao modo asilar.

No que se refere à categoria médica, nesta investigação, percebe-se que os profissionais da SF delegam ao saber médico o poder de atender as crises, pela possibilidade de prescrição de medicamentos. Ressalta-se que para a realização desta pesquisa foram convidados todos os profissionais de nível superior da SF e do CAPS, porém, não houve nenhum representante da categoria médica. Isso pode ser entendido como uma sobrecarga destes profissionais associada a um cuidado ainda um tanto médico centrado, em que a presença deste profissional é tida pela população e pelos demais membros da equipe como primordial para a atenção em saúde. Dessa forma, este estudo se depara com o questionamento sobre como envolver mais o profissional médico nas discussões coletivas, no cuidado em saúde mental e no apoio matricial.

Equipes de SF fortalecidas nos atributos da integralidade, longitudinalidade, acesso e coordenação do cuidado não deveriam simplesmente encaminhar os pacientes em crise ou negligenciá-los. Se assim o fizerem, descumprem sua função na rede de saúde. É importante para um paciente e para sua família saber que pode contar com sua equipe de SF nesses momentos cruciais da vida e é de grande valor para a equipe atender os pacientes na crise, entendendo melhor cada um, nos diferentes momentos de sua existência.

Entende-se que a responsabilidade da atenção dessas situações complexas não é somente da SF, sendo fundamental a articulação com serviços especializados em saúde mental, como o CAPS III, que ofertam cuidado intensivo, acolhimento noturno, necessário nas crises graves. Mas, o atendimento a algumas crises mais brandas, o acolhimento e algumas abordagens das crises mais graves podem ser feitas pelas equipes de SF, a fim da manutenção do vínculo e da realização de encaminhamentos responsabilizados.

Para isso, acredita-se ser importante abrir o debate em torno da importância da APS no que se refere a esse tema, contribuindo com a reflexão sobre um dos nós da Reforma Psiquiátrica Brasileira: a clínica da crise. Ainda, no que tange aos serviços pesquisados no presente estudo, destacamos que por se tratar de um CAPS III, esse serviço pode funcionar como um dispositivo para auxiliar na discussão sobre as abordagens possíveis da SF na crise. Mas para tal é necessário uma agenda de apoio matricial e uma atitude ativa por parte do CAPS no que se refere à proposição desta pauta. 
Barbier, R. (2002). A pesquisa-ação. Brasília: Plano Editora. Bardin, L. (1977). Análise de Conteúdo. Lisboa: Edições 70.

Basaglia, F. et al. (1991). A loucura na sala de jantar. São Paulo: Resenha.

Brasil. Ministério da Saúde (2004). Secretaria de Atenção à Saúde. Departamento de Ações Programáticas Estratégicas. Saúde mental no SUS: os centros de atenção psicossocial. Brasília.

Brasil. Ministério da Saúde (2011). Gabinete Ministerial. Portaria n. 2.488, de 21 de outubro de 2011. Aprova a Política Nacional de Atenção Básica. Diário Oficial da União, Brasília, DF, 24 out. 2011. Seção 1, p. 48-55.

Campos, G. W. S. (1999). Equipes de referência e apoio especializado matricial: um ensaio sobre a reorganização do trabalho em saúde. Ciência \& Saúde Coletiva, Rio de Janeiro, Abrasco, 4(2), 393-403.

Campos, G. W. S. \& Domitti, A. C. (2007, fevereiro). Apoio Matricial e equipe de referência: uma metodologia para a gestão do trabalho interdisciplinar em saúde. $\mathrm{Ca}$ dernos de Saúde Pública, Rio de Janeiro, 23(2), 399-407.

Carvalho, N. R. de \& Costa, I. I. (2008). Primeiras crises psicóticas: identificação de pródromos por pacientes e familiares. Psicologia Clínica, Rio de Janeiro, 290(1), 153-164.

Costa, I. I. (2006). Adolescência e a primeira crise psicótica: problematizando a continuidade entre o sofrimento psíquico normal e o psíquico grave. Anais do II Congresso Internacional de Psicopatologia Fundamental. Recuperado em 10 de novembro de 2012, de: <http://www.fundamentalpsychopathology.org/anais2006/4.69.3.1.htm>.

Costa-Rosa, A. (2000). O modo psicossocial: um paradigma das práticas substitutivas ao modo asilar. In P. Amarante (Org.). Ensaios: subjetividade, saúde mental, sociedade. Rio de Janeiro: Fiocruz.

Franco, M. L. P. B. (2008). Análise de conteúdo. Brasília: Liber Livro Editora.

Lobosque, A. M. (2007). Aulas de Saúde Mental para Equipes do Programa de Saúde da Família. Coordenadoria de Educação Permanente. Escola de Saúde Pública do Estado de Minas Gerais. Recuperado em 10 de novembro de 2012, de: < http:// espacosaudemental.esp.mg.gov.br/arquivos/aulas-de-saude-mental-para-equipesdo-programa-de-saude-da-familia/>.

Nicácio, F. \& Campos, G. W. S. (2004, maio/agosto). A complexidade da atenção às situações de crise: contribuições da desinstitucionalização para a invenção de práticas inovadoras em saúde mental. Revista de Terapia Ocupacional, São Paulo, 15(2), 71-81.

Onocko Campos, R. T. (2001, maio/agosto). Clínica: a palavra negada: sobre as práticas clínicas nos serviços substitutivos de Saúde Mental. Saúde em Debate, Rio de Janeiro, 25(58), 98-111. 
Onocko Campos, R. T. \& Gama, C. (2010). Saúde Mental na Atenção Básica. In G. W. S. Campos \& A. V. P. Guerreiro. Manual de práticas de atenção básica: saúde ampliada e compartilhada. São Paulo: Hucitec.

Thiollent, M. (1996). Metodologia da pesquisa-ação. 7. ed. São Paulo: Cortez.

\section{Resumos}

(Matrix support in mental health: strengthening family health in clinical work with crisis situations)

The objective of this paper is to discuss matrix support in mental health provided to family health teams, and the relation of this type of support to crisis situations in mental health. This is done through the analysis of action research conducted on family health teams and on work carried out at a mental health treatment center (CAPS III) in the Rocinha slum neighborhood of Rio de Janeiro. Professionals from different areas participated in operative discussion groups and the resulting data were examined through content analysis. The importance of involving family health teams in clinical work with crisis situations is highlighted.

Key words: Mental health services, primary health care, matrix support, crisis

(Support matriciel en santé mentale: renforcer la santé de la famille dans la clinique de la crise)

Cet article analyse l'appui matriciel en santé mentale qui est donné aux équipes de Santé de la Famille (SF) et son rapport avec les situations de crise en santé mentale. Il s'agit d'une recherche-action menée avec les équipes de SF et un Centre de Soins Psychosocial (CAPS III) dans la Favela Rocinha, à Rio de Janeiro. Des professionnels de différentes catégories ont participé à de groupes opérationnels de réflexion et les données ont été examinées par moyen d'une analyse de contenu. On souligne l'importance d'intégrer la SF dans la clinique de la crise.

Mots clés: Services de Santé Mental, soins de santé primaires, support matriciel, crise

(Soporte matricial en salud mental: fortalecimiento de la salud de la familia en la clínica de crisis).

El objetivo es abordar el (al) soporte matricial en salud mental a los equipos de Salud de la Familia (SF) y su relación con las situaciones de crisis en salud mental. Se trata de una investigación-acción llevada a cabo con equipos de SF y un CAPS III en Rocinha, Rio de Janeiro. Participaron profesionales de diferentes categorías en grupos operacionales de reflexión (en) y los datos han sido examinados por el método 


\section{SAÚDE MENTAL}

de análisis del contenido. Es destacada la importância de la participación de la SF con respecto a la clínica de crisis.

Palabras clave: Servicios de Salud Mental, atención primaria de salud, soporte matricial, crisis

(Elterliche Unterstützung im Rahmen der geistigen Gesundheit: Stärkung der Gesundheit der Familie in der Krisenklinik)

Das Ziel ist, die Unterstützung des Mitarbeiterteams der Organisation Saúde da Família (SF) (Gesundheit der Familie) durch die Eltern im Rahmen der geistigen Gesundheit und ihren Zusammenhang mit der Krise im Bereich der geistigen Gesundheit anzusprechen. Dies ist eine Forschungsinitiative des Mitarbeiterteams der Organisation SF und eines CAPS III (Centro de Atendimento Psicossocial / Psychosoziales Betreuungszentrum) im Slum Rocinha, Rio de Janeiro. Es haben Experten aus verschiedenen Berufskategorien an operativen Reflexionsgruppen teilgenommen und die Daten anhand einer Inhaltsanalyse untersucht. Im Beitrag wird hervorgehoben, wie wichtig es ist die Organisation SF in Angelegenheiten der Krisenklinik miteinzubeziehen.

Schlüsselwörter: Psychosoziale Dienstleistung, Primäre Gesundheitsversorgung, elterliche Unterstützung, Krise

Citação/Citation: Minozzo, F. \& Costa, I. I. de (2013, setembro). Apoio matricial em saúde mental: fortalecendo a saúde da família na clínica da crise. Revista Latinoamericana de Psicopatologia Fundamental, 16(3), 438-450.

Editor do artigo/Editor: Profa. Dra. Ana Cristina Costa de Figueiredo e Profa. Dra. Andrea Máris Campos Guerra 
Recebido/Received: 17.1.2013/ 1.17.2013 Aceito/Accepted: 25.3.2013/3.25.2013

Copyright: (C) 2009 Associação Universitária de Pesquisa em Psicopatologia Fundamental/ University Association for Research in Fundamental Psychopathology. Este é um artigo de livre acesso, que permite uso irrestrito, distribuição e reprodução em qualquer meio, desde que o autor e a fonte sejam citados / This is an open-access article, which permits unrestricted use, distribution, and reproduction in any medium, provided the original author and source are credited.

Financiamento/Funding: Os autores declaram não ter sido financiados ou apoiados / The authors have no support or funding to report.

Conflito de interesses/Conflict of interest: Os autores declaram que não há conflito de interesses / The authors declare that has no conflict of interest.

\section{Fabiane Minozzo}

Mestre em Psicologia Clínica e Cultura pela Universidade de Brasília - UnB (Brasília, DF, Br); Especialista em Saúde Mental pela mesma Universidade; Especialista em Atenção Primária à Saúde - modalidade residência multiprofissional pela Escola de Saúde Pública do Rio Grande do Sul - ESP/RS (Porto Alegre, RS, Br); Psicóloga.

Rua Antonio Parreiras, 25/606 - Ipanema

22411-020 Rio de Janeiro, RJ, Br.

Fone: (21) 8016-2562

e-mail: fabiminozzo@yahoo.com.br

\section{ILENO IzÍDIO DA COSTA}

Doutor em Psicologia Clínica pela Universidade de Brasília - UnB/Warwick (Brasília, DF, Br); Professor Adjunto do Departamento de Psicologia Clínica da Universidade de Brasília - UnB (Brasília, DF, Br); MA em Filosofia e Ética da Saúde Mental (Warwick/Inglaterra); Coordenador do Grupo de Intervenção Precoce nas Psicoses - GIPSI (Brasília, DF, Br); Presidente da Associação de Saúde Mental do Cerrado - Ascer (Brasília, DF, Br).

ICC Sul, Campus Universitário Darcy Ribeiro 70910-900 Brasília, DF, Br.

Fone: (61) 9981-3287

e-mail: ileno@unb.br 